Eixo Temático: Biologia Aplicada

\title{
ET-09-016 \\ OTIMIZAÇÃO DO PROCESSO DE REMOÇÃO DO CORANTE REMAZOL BLACK B POR USO DE BIOMASSA MISTA DE Aspergillus niger E CAPIM ELEFANTE (Pennisetum purpureum Schum)
}

Iranildo José da Cruz Filho ${ }^{1}$, Leticia de Paula Silva de Oliveira ${ }^{2}$, Hanna Katarina Lopes Ferreira ${ }^{1}$, Suellen Emilliany Feitosa Machadoํㅡㄹ Larissa Ribeiro Souza ${ }^{1}$, Valmir Felix de Lima ${ }^{2}$, Agrinaldo Jacinto do Nascimento Junior ${ }^{3}$, Samyla Kelly Gomes da Silva ${ }^{1}$, Olga Martins Marques $^{2}$

${ }^{1}$ Universidade Federal de Pernambuco (UFPE), Departamento de Antibióticos, Pernambuco.

${ }^{2}$ Universidade Federal de Pernambuco (UFPE). Departamento de Engenharia Química, Pernambuco.

${ }^{3}$ Instituto Federal do Paraná (IFPR), Paraná.

http://dx.doi.org/10.21472/congrebio2016.et-09-016

\section{RESUMO}

O setor têxtil produz um efluente bastante tóxico, com uma grande concentração de corantes, os quais são envolvidos no processo de tingimento. Parte deles são eliminados na natureza causando danos a fauna e a flora. Diante dessa problemática, metodologias têm sido desenvolvidas afim de tratar esses efluentes antes de serem lançados na natureza. Dentre essas está a biossorção, tecnologia que utiliza micro-organismos vivos ou mortos para remoção de contaminantes. A biossorção se torna bastante atrativa devido aos bons resultados e a fácil obtenção de micro-organismos, já que muitos deles são facilmente isolados e cultivados. Uma espécie alvo de pesquisas em diversos processos industriais devido a sua grande produção de enzimas e produtos de interesse industrial é o Aspergillus niger, um fungo filamentoso largamente encontrado no solo, podendo crescer em diversas culturas vegetais. Neste trabalho foi utilizado como biossorvente a biomassa de Aspergillus niger crescida em capim elefante por $72 \mathrm{~h}$. A massa produzida foi autoclavada a $121^{\circ} \mathrm{C}$ por $30 \mathrm{~min}$, em seguida filtrada lavada e seca a $65^{\circ} \mathrm{C}$ até base seca por $72 \mathrm{~h}$. Com as massas produzidas, foi realizado um planejamento fatorial $2^{5}$ com o objetivo de estudar o efeito das interações: $\mathrm{pH}$, agitação, temperatura, concentração de corante e biomassa. A taxa de remoção foi obtida como resposta a essas interações. Os resultados do estudo permitiram identificar que os fatores principais temperatura e agitação não mostraram significância estatística numa margem de 95\% de confiança. Deve-se destacar ainda, que tanto o fator linear $\mathrm{pH}$ quanto o da concentração de biomassa ocasionaram efeitos negativos na biossorção do corante, mostrando que tanto o aumento da basicidade do meio quanto o aumento da concentração de biomassa utilizada diminui o processo biossortivo. A melhor condição obtida no planejamento foi pH igual a 2,0; temperatura de $50{ }^{\circ} \mathrm{C}$; agitação de 150 rpm; concentração de biomassa de 0,5 g e concentração inicial de corante de 25 mg. $\mathrm{L}^{-1}$, ocasionando uma remoção de $91,78 \%$.O estudo permitiu verificar que utilização de biomassa fúngica associada com vegetal pode se tornar uma nova metodologia para tratamento de efluentes têxteis.

Palavras-chave: Biossorção; Capim elefante; Aspergillus niger; Tratamento de efluentes têxteis. 


\section{INTRODUÇÃO}

O crescimento econômico do setor têxtil no Brasil tem trazido melhores condições de vida à população, gerando novos empregos e qualificando mão de obra. Entretanto esse setor tem lançado nos efluentes hídricos compostos químicos resultantes do processo de tingimento, os corantes têxteis, os quais são um dos maiores causadores de problemas ambientais (KUNZ et al., 2002).

Os corantes têxteis utilizados atualmente são compostos orgânicos sintéticos, cuja finalidade é conferir coloração a determinadas fibras, sejam elas sintéticas ou naturais. Apresentam em sua estrutura dois componentes principais: o grupo cromóforo, conjunto de átomos que absorvem a radiação e confere coloração a fibra, e o grupo funcional, que permite a fixação na fibra dos tecidos (CARDOSO et al., 2011).

Esses compostos podem ser classificados de acordo com sua estrutura química e pelo método de fixação à fibra têxtil. Dentre os mais utilizados pela indústria têxtil estão os corantes reativos do tipo azóico, caracterizados quimicamente pela presença de, pelo menos, um agrupamento azo $(-\mathrm{N}=\mathrm{N}-)$, formando uma ligação química bastante estável com a celulose (KUNZ et al., 2002). Os produtos submetidos a tingimentos com corantes reativos apresentam colorações mais resistentes a água e ao sabão, isto devido à ligação covalente formada entre os grupos reativos e as moléculas da fibra do tecido (DALLAGO e SMANIOTTO, 2005). São conhecidos cerca de 10 mil tipos de corantes e mundialmente são produzidas 700 mil toneladas. Estima-se que mais de 20 mil toneladas destes produtos são anualmente consumidas pelas indústrias do setor têxtil (DALLAGO e SMANIOTTO, 2005).

A contaminação de rios e lagos por estes compostos, além de conferir cor, causa diversos danos à fauna e a flora, pois, a presença dessas substâncias impossibilita a passagem da luz solar, diminuindo a atividade fotossintética natural, reduzindo assim a oxigenação da água e ocasionando possíveis desequilíbrios ecológicos, além de tornar a água imprópria para o consumo humano (BRASIL, 2011). Diante deste problema, tecnologias têm sido desenvolvidas ou otimizadas, visando a uma melhor maneira de remover a cor dos efluentes têxteis, o que proporciona proteção ao meio ambiente e controle da poluição. Segundo a Resolução CONAMA $n^{\circ} 430 / 2011$ (BRASIL, 2011) os efluentes de qualquer fonte poluidora somente poderão ser lançados, direta ou indiretamente, nos corpos de água após o devido tratamento, e que não apresente efeitos tóxicos.

Uma das tecnologias bastante utilizadas devido ao baixo custo e a reutilização de materiais é a biossorção. Este fenômeno físico-químico consiste na concentração espontânea de determinadas espécies (contaminantes) na superfície de um material (espécie biológica). É um processo passivo, rápido, reversível e independente de energia metabólica, realizado tanto por biomassa viva quanto por biomassa morta (KYZAS et al., 2012). Dentre os mecanismos envolvidos no processo biossortivo, destacam-se troca iônica, complexação, precipitação e cristalização.

Apesar de recente quando comparado a outros sistemas biológicos, a utilização de fungos na remoção de poluentes tem sido bastante relatada na literatura. Por exemplo, algumas espécies de Aspergillus são conhecidas por adsorver corantes e também por utilizar esses componentes como substrato nutritivo, transformando-os em compostos não tóxicos ou de baixa toxicidade (BISHNOI e GARIMA, 2005).

De acordo com Bishnoi e Garima (2005) as paredes dos fungos contêm grandes quantidades de polissacarídeos e proteínas, as quais possuem diversos grupos funcionais, tais como carboxilas, hidroxilas, sulfatos, fosfatos e grupos amina, capazes remover contaminantes. Dessa forma esses microrganismos podem se enquadrar como bons adsorventes no tratamento de efluentes. 


\section{OBJETIVO}

Avaliar a remoção do corante Remazol Black B em soluções líquidas, preparadas em laboratório, pelo uso da biomassa mista de Aspergillus niger e capim elefante (Pennisetum Purpureum Schum) como biossorvente.

\section{METODOLOGIA}

\section{Corante}

O corante utilizado neste experimento foi o Remazol Black B (RBB), cuja massa e a forma molecular são $991,816117 \mathrm{~g} \cdot \mathrm{mol}^{-1}$ e $\mathrm{C}_{26} \mathrm{H}_{21} \mathrm{~N}_{5} \mathrm{Na}_{4} \mathrm{O}_{19} \mathrm{~S}_{6}$, respectivamente. Ele apresenta seu comprimento de onda máximo de absorção na faixa de $597 \mathrm{~nm}$.

Curva analítica do corante RBB. A concentração residual do corante na solução após o processo de adsorção foi avaliada através da leitura da absorbância a $597 \mathrm{~nm}$ a $30{ }^{\circ} \mathrm{C}$ e convertida em $\mathrm{mg} \cdot \mathrm{L}^{-1}$ através de uma curva analítica na faixa de 5 a $30 \mathrm{mg} \cdot \mathrm{L}^{-1}$. Essa curva é baseada na Lei de Lambert-Beer de acordo com a equação 1.

$A=\varepsilon b c$

Onde: A é a absorbância, $\varepsilon$, absortividade molar $\left(\mathrm{mL} \cdot \mathrm{mg}^{-1} \cdot \mathrm{cm}^{-1}\right)$, c é a concentração $\left(\mathrm{mg} \cdot \mathrm{L}^{-1}\right)$ e b é o caminho ótico (parte lateral da cubeta $=1 \mathrm{~cm}$ ).

\section{Produção do biossorvente misto}

Capim Elefante (Pennisetum purpureum). O capim elefante (Pennisetum purpureum Schum) utilizado neste trabalho, foi coletado em um canteiro, específico da espécie, pertencente ao Departamento de Zootecnia, da Universidade Federal Rural de Pernambuco (UFRPE). O material foi cortado em pedaços, lavado e posto para secar a $75{ }^{\circ} \mathrm{C}$, por $72 \mathrm{~h}$. Em seguida, foi triturado em moinho de faca, tamisado em peneiras de Tyler $(1,43 \mathrm{~mm})$, armazenado em saco plástico vedado e mantido na temperatura ambiente.

Aspergillus niger. Foi utilizada a cultura pura do fungo Aspergillus niger (ATCC 1015), pertencente à Coleção de Microrganismos, do Laboratório de Microbiologia Industrial, do Departamento de Engenharia Química (DEQ), da Universidade Federal de Pernambuco (UFPE). A cultura foi previamente cultivada por 5 dias, a $30^{\circ} \mathrm{C}$, em tubos contendo $10,0 \mathrm{~mL}$ de caldo Czapeck (CZ), conforme a composição a seguir, em g. $\mathrm{L}^{-1}: \mathrm{NaNO}_{3}(3,0) ; \mathrm{K}_{2} \mathrm{HPO}_{4}(1,0)$; $\mathrm{MgSO}_{4}(0,50) ; \mathrm{KCl}(0,5) ; \mathrm{FeSO}_{4}(0,01)$; Sacarose $(30,0)$.

Após este período, os conteúdos dos tubos foram transferidos para frascos de Roux de capacidade de $500 \mathrm{~mL}$, contendo $200 \mathrm{~mL}$ do mesmo meio solidificado com $16 \mathrm{~g} \cdot \mathrm{L}^{-1}$ de agar. Estes foram mantidos a $30^{\circ} \mathrm{C}$, por 5 dias, e após esse tempo os esporos do fungo foram removidos e postos em suspensão aquosa. Avaliou-se a concentração celular da solução usandose a técnica de contagem de esporos pelo uso de uma câmara de Neubauer, ajustou-se a uma concentração de $10^{7}$ esporos $\cdot \mathrm{mL}^{-1}$.

Biossorvente misto (capim elefante e fungo). Um volume de 5,0 mL de suspensão de esporos foi transferido para um frasco de Erlenmeyer previamente esterilizado contendo $250 \mathrm{~mL}$ de caldo Czapec Modificado, tendo como fonte de carbono 67\% de capim elefante e $33 \%$ de sacarose. O sistema foi mantido sob agitação de $200 \mathrm{rpm}$ a $30{ }^{\circ} \mathrm{C}$, por $72 \mathrm{~h}$. Todo processo foi realizado em condições assépticas. A biomassa produzida foi autoclavada a $121^{\circ} \mathrm{C}$ durante 30 minutos, filtrada e em seguida lavada com água estéril e seca a $65^{\circ} \mathrm{C}$, por 72 horas.

Planejamento Experimental. Para analisar os efeitos de variáveis significativas na biossorção do corante no biossorvente misto de capim elefante com o fungo, foi utilizado o 
método de Planejamento Fatorial com três variáveis em dois níveis $\left(2^{5}\right)$ mais o ponto central em triplicata, totalizando 35 ensaios em duplicata, realizados de forma aleatória.

$\mathrm{O}$ pH, temperatura, agitação, concentração de biossorvente e concentração de corante foram as variáveis independentes (fatores) e, a porcentagem de remoção do corante foi a variável dependente (resposta). Os ensaios foram realizados em mesa agitadora num período de tempo de $1 \mathrm{~h}$. As soluções foram ajustadas com $\mathrm{NaOH}$ e/ou $\mathrm{HCl}$ 3,0 M até obter-se o pH desejado. Posteriormente, alíquotas foram retiradas e centrifugadas a $5000 \mathrm{rpm}$ por $3 \mathrm{~min}$. A fração do sobrenadante foi analisada por espectrofotometria UV/Vis em $\lambda=597 \mathrm{~nm}$. O percentual de remoção foi calculado usando-se a Equação 2:

$$
\mathrm{R}=\frac{100\left(\mathrm{C}_{0}-\mathrm{C}_{\mathrm{f}}\right)}{\mathrm{C}_{0}}
$$

Onde: $\mathrm{C}_{0}$ é a concentração inicial de corante RBB $\left(\mathrm{mg} \cdot \mathrm{L}^{-1}\right)$; $\mathrm{C}_{\mathrm{f}}$ é a concentração de corante RBB no tempo de equilíbrio $\left(\mathrm{mg} \cdot \mathrm{L}^{-1}\right)$.

Todos os ensaios realizados seguiram a matriz fatorial mostradas pelas Tabelas 1 e 2 e as análises foram realizadas através do software Statistica ${ }^{\circledR}$ 8.0.

Tabela 1. Matriz fatorial $2^{5}$ decodificada.

\begin{tabular}{l|c|c|c|c|c}
\hline $\begin{array}{l}\text { Níveis } \\
\text { decodificados }\end{array}$ & $\mathbf{p H}$ & $\begin{array}{c}\text { Temperatura } \\
\left({ }^{\circ} \mathbf{C}\right)\end{array}$ & $\begin{array}{c}\text { Agitação } \\
\mathbf{( r p m )}\end{array}$ & $\begin{array}{c}\text { Concentração de } \\
\text { Biossorvente (g) }\end{array}$ & $\begin{array}{c}\text { Concentração } \\
\text { de Corante } \\
\left(\mathbf{m g} \cdot \mathbf{L}^{-\mathbf{1}} \mathbf{)}\right.\end{array}$ \\
\hline-1 & 2,0 & 30 & 150 & 0,5 & 25 \\
\hline 0 & 3,5 & 40 & 200 & 1,5 & 55 \\
\hline+1 & 5,0 & 50 & 250 & 2,5 & 85 \\
\hline
\end{tabular}

Tabela 2. Matriz fatorial $2^{5}$ codificada.

\begin{tabular}{l|c|c|c|c|c}
\hline Ensaio & pH & Temperatura & Agitação & $\begin{array}{c}\text { Concentração de } \\
\text { Biossorvente }\end{array}$ & $\begin{array}{c}\text { Concentração de } \\
\text { Corante }\end{array}$ \\
\hline $\mathbf{1}$ & + & + & + & + & + \\
\hline $\mathbf{2}$ & - & + & + & + & + \\
\hline $\mathbf{3}$ & + & - & + & + & + \\
\hline $\mathbf{4}$ & - & - & + & + & + \\
\hline $\mathbf{5}$ & + & + & - & + & + \\
\hline $\mathbf{6}$ & - & + & - & + & + \\
\hline $\mathbf{7}$ & + & - & - & + & + \\
\hline $\mathbf{9}$ & - & - & - & + & + \\
\hline $\mathbf{1 0}$ & + & + & + & - & + \\
\hline $\mathbf{1 1}$ & - & + & + & - & + \\
\hline $\mathbf{1 2}$ & - & - & + & - & + \\
\hline $\mathbf{1 3}$ & + & - & + & - & + \\
\hline $\mathbf{1 4}$ & - & + & - & - & + \\
\hline $\mathbf{1 6}$ & + & - & - & - & - \\
\hline $\mathbf{1 7}$ & + & - & - & - & + \\
\hline
\end{tabular}


Tabela 2. Continuação.

\begin{tabular}{l|c|c|c|c|c}
\hline Ensaio & pH & Temperatura & Agitação & $\begin{array}{c}\text { Concentração de } \\
\text { Biossorvente }\end{array}$ & $\begin{array}{c}\text { Concentração de } \\
\text { Corante }\end{array}$ \\
\hline $\mathbf{1 8}$ & - & + & + & + & - \\
\hline $\mathbf{1 9}$ & + & - & + & + & - \\
\hline $\mathbf{2 0}$ & - & - & + & + & - \\
\hline $\mathbf{2 1}$ & + & + & - & + & - \\
\hline $\mathbf{2 2}$ & - & + & - & + & - \\
\hline 23 & + & - & - & + & - \\
\hline $\mathbf{2 4}$ & - & - & - & + & - \\
\hline $\mathbf{2 5}$ & + & + & + & - & - \\
\hline $\mathbf{2 6}$ & - & + & + & - & - \\
\hline 27 & + & - & + & - & - \\
\hline $\mathbf{2 8}$ & - & - & + & - & - \\
\hline 29 & + & + & - & - & - \\
\hline 30 & - & + & - & - & - \\
\hline 31 & + & - & - & - & - \\
\hline 32 & - & - & - & - & $\mathbf{0}$ \\
\hline 33 & $\mathbf{0}$ & $\mathbf{0}$ & $\mathbf{0}$ & $\mathbf{0}$ & $\mathbf{0}$ \\
\hline 34 & $\mathbf{0}$ & $\mathbf{0}$ & $\mathbf{0}$ & $\mathbf{0}$ & $\mathbf{0}$ \\
\hline 35 & $\mathbf{0}$ & $\mathbf{0}$ & $\mathbf{0}$ & $\mathbf{0}$ & - \\
\hline
\end{tabular}

\section{RESULTADOS E DISCUSSÃO}

\section{Planejamento experimental}

O planejamento fatorial foi avaliado através do programa Statistica ${ }^{\circledR}$ (versão 8.0). Na Tabela 3 encontram-se os resultados dos efeitos dos fatores na resposta, isto é, como as condições experimentais interferem na variável dependente, que nesse caso, é o percentual de remoção do corante. Os valores que estão em negrito são os efeitos estatisticamente significativos para a biossorção do RBB pela biomassa mista de Aspergillus niger e capim elefante (Pennisetum purpureum shum).

Tabela 3. Efeitos dos fatores pH, temperatura, agitação, concentração de biomassa e de corante na biossorção em biomassa mista de fungo e capim.

\begin{tabular}{ll}
\hline Efeitos & Estimativa \pm Erro padrão \\
\hline Média Global & $\mathbf{1 4 , 5 1 0} \pm \mathbf{6 , 6 6 9}$ \\
Efeitos Principais & \\
(1) $\mathbf{p H}(\mathrm{L})$ & $\mathbf{- 6 5 , 8 4 6} \pm \mathbf{4 , 0 8 4}$ \\
pH (Q) & $\mathbf{3 6 , 5 0 3} \pm \mathbf{1 3 , 9 4 9}$ \\
(2) Temperatura (L) & $0,168 \pm 4,084$ \\
(3) Agitação (L) & $-2,719 \pm 4,084$ \\
(4) Concentração de Biomassa (L) & $\mathbf{- 1 1 , 7 8 2} \pm \mathbf{4 , 0 8 4}$ \\
(5) Concentração de Corante (L) & $\mathbf{1 0 , 4 1 8} \pm \mathbf{4 , 0 8 4}$ \\
\hline
\end{tabular}


Tabela 3. Continuação.

\begin{tabular}{cc}
\hline Efeitos de interação de dois fatores \\
(1) e (2) \\
(1) e (3) & $2,535 \pm 4,084$ \\
(1) e (4) & $5,942 \pm 4,084$ \\
(1) e (5) & $3,441 \pm 4,084$ \\
(2) e (3) & $\mathbf{1 6 , 0 7 0} \pm \mathbf{4 , 0 8 4}$ \\
(2) e (4) & $\mathbf{- 1 2 , 6 3 6} \pm \mathbf{4 , 0 8 4}$ \\
(2) e (5) & $-5,659 \pm 4,084$ \\
(3) e (4) & $4,440 \pm 4,084$ \\
(3) e (5) & $-5,435 \pm 4,084$ \\
(4) e (5) & $\mathbf{- 1 5 , 5 9 2} \pm \mathbf{4 , 0 8 4}$ \\
\end{tabular}

Nota-se, de acordo com a Tabela 3, que os efeitos principais temperatura e agitação não foram significativos no intervalo estabelecido pelo planejamento. Já, todos os outros fatores, mostraram significância estatística numa margem de 95\% de confiança.

Deve-se destacar ainda, que tanto o fator linear $\mathrm{pH}$ quanto o da concentração de biomassa ocasionaram efeitos negativos na biossorção do corante, mostrando que tanto o aumento da basicidade do meio quanto o aumento da concentração de biomassa utilizada diminui o processo biossortivo do corante-biossorvente.

Pelos resultados apresentados no planejamento observa-se que em pHs mais ácidos temse uma melhor performance do material, isso porque a biossorção de corantes é influenciada pela carga superficial do material e pelo grau de ionização do corante. Como o valor do $\mathrm{pH}$ da solução influencia significativamente a eficiência do processo, a variação deste fator faz com que o biossorvente apresente superfície positiva favorecendo a biossorção de ânions ou de cargas negativa (KUMAR e BARAK, 2013).

Em um efluente contaminado pelo RBB, a molécula do corante apresenta alguns grupos funcionais passíveis de protonação devido a diminuição do $\mathrm{pH}$, mesmo assim, o grupo sulfônico preserva a sua forma aniônica já que seu pKa é inferior a 1. Essa interação de cargas (positivas e negativas) sugere que o processo biossortivo ocorra via grupos protonados do material com grupos aniônicos do corante (CARDOSO et al., 2011).

Com o aumento do $\mathrm{pH}$ da solução, embora o corante permaneça na forma aniônica, há uma diminuição de sítios protonados na superfície do biossorvente ocasionando a diminuição da capacidade biossortiva devido ao aumento e repulsão de cargas negativas (KUMAR e BARAK, 2013).

Apesar da temperatura e agitação não terem sido significantes na sua forma principal, o efeito da interação destes fatores foi significativo de forma prejudicial para o resultado, pois, o aumento simultâneo destes fatores ocasionou em média, um decréscimo de pouco mais de $12 \%$ na remoção do corante. Essas duas variáveis quando aumentadas, normalmente, tendem a provocar um acréscimo na energia cinética e na mobilidade das moléculas do sistema o que favorece o aumento na taxa de difusão intrapartícula do corante (KUMAR e BARAKAT, 2013). No entanto, quando estes parâmetros tornam-se muito elevados eles podem favorecer o processo contrário, o de dessorção (NETPRADIT et al., 2004).

Em estudos realizados por Snirivasan e Viraraghavan (2010) com algas, foi verificado que altas temperaturas podem causar danos à superfície do biossorvente, diminuindo a biossorção, além de provocar uma maior solubilização do contaminante em solução, diminuindo assim o número de interações adsorbato-biossorvente. Estes fatos, porém, não foram verificados dentro do limite do planejamento, que foi de até $50^{\circ} \mathrm{C}$. Isto demostra que o que veio causar o decréscimo na interação foi a utilização de um valor alto na agitação, pois, segundo Kyzas et al. (2012) em estudos realizados com resíduo industrial de café, foi verificado que em agitações 
mais baixas (60-80 rpm) de processo tem-se uma melhor capacidade de remoção e que acima desses valores não houve qualquer aumento na remoção, esses resultados corroboram com aqueles obtidos no planejamento onde em agitações inferiores a $150 \mathrm{rpm}$ se tem um aumento na capacidade de remoção.

Segundo Tavlieva et al. (2013) aumentando a massa de biossorvente aumenta-se a área de superfície para biossorção e, consequentemente, a taxa de remoção também sofre elevação. Porém, esse resultado contradiz o resultado obtido no planejamento onde teve-se melhor remoção em concentrações menores de biossorvente. Esse fato é devido a liberação de compostos que conferem cor esverdeada provenientes de resquícios de clorofila presentes na biomassa fresca, liberados na solução, diminuindo o processo. Além da liberação de compostos, em concentrações muito altas de biomassas pode ocorrer a competição entre si, partículas do biossorvente ligando-se a uma mesma molécula de corante, diminuindo assim os sítios de biossorção reduzindo a capacidade de remoção (SRINIVASAN e VIRARAGHAVAN, 2010).

As melhores condições experimentais de remoção do corante RBB na biomassa mista de capim elefante e do fungo Aspergillus niger foram encontradas em $\mathrm{pH}$ igual a 2,0, numa temperatura de $50{ }^{\circ} \mathrm{C}$, agitação de $150 \mathrm{rpm}$, concentração de biomassa de 0,5 g e concentração inicial de corante de $25 \mathrm{mg} \cdot \mathrm{L}^{-1}$, ocasionando uma remoção de $91,78 \%$.

A
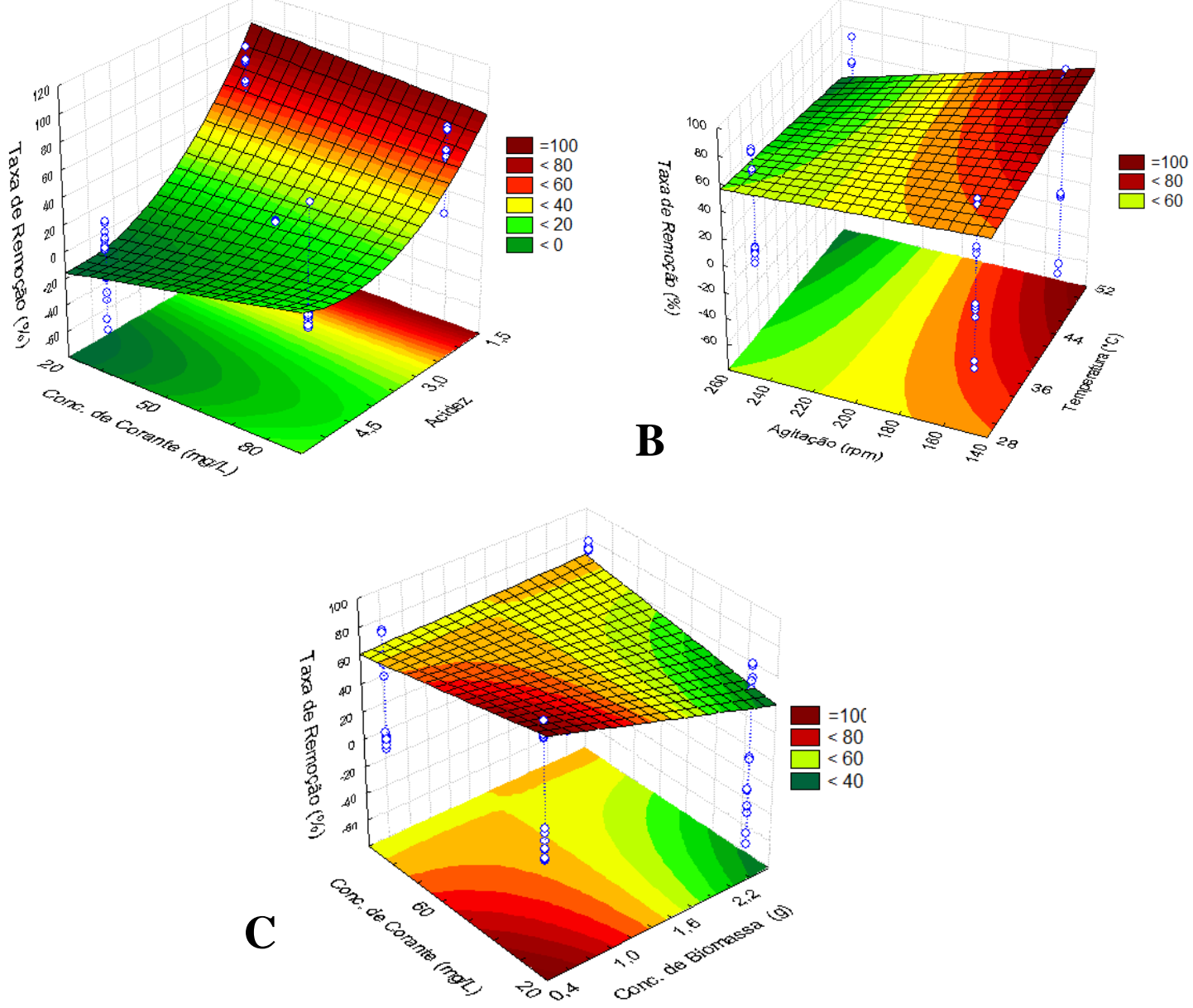

Figura 1. Superfícies de resposta das interações: A) (1) pH e (5) Concentração de corante (mg. $\mathrm{L}^{-1}$ ); B) (2) Temperatura $\left({ }^{\circ} \mathrm{C}\right)$ e (3) Agitação (rpm); C) (4) Concentração de Biomassa (g) e (5) Concentração de corante (mg.L ${ }^{-1}$ ). 
A Figura 1 apresenta as superfícies de resposta obtidas pelo software, onde se pôde obter otimizações das condições experimentais, de forma a alcançar teoricamente taxas de $100 \%$ de remoção.

A curva da interação do $\mathrm{pH}$ e da temperatura, mostrada pela Figura 1A, indica que os valores de maior remoção, caracterizados pela coloração vermelha, são obtidos na região de $\mathrm{pH}$ inferior a 2,0. De acordo com a Figura $1 \mathrm{~B}$ nota-se que a temperatura em torno dos $50{ }^{\circ} \mathrm{C}$ e uma agitação baixa (menor que $150 \mathrm{rpm}$ ) favoreceriam o processo ainda mais, obtendo melhores taxas de remoção. Esse aumento na temperatura proporcional ao aumento da capacidade biossortiva evidencia que provavelmente tem-se um processo endotérmico e vem retificar a afirmativa que no nível mais alto $(+1)$ escolhido no planejamento para a temperatura, o valor ainda ocasiona benefício, não sendo considerado alto o suficiente para ocasionar a perda das funções do adsorvente, evidenciando que o decréscimo mostrado pela interação dos fatores foi devido ao aumento da agitação, que mostrou ser muito desfavorável no processo.

A superfície de resposta obtida pela interação da concentração de biomassa e da concentração de corante mostrou que valores de concentrações menores que $0,5 \mathrm{~g}$ e $25 \mathrm{mg} \cdot \mathrm{L}^{-1}$ respectivamente ocasionariam uma remoção de $100 \%$ do RBB da solução. Para soluções mais diluídas, a taxa de captação inicial do corante é mais rápida e um aumento na concentração inicial da solução faz com que o processo se torne mais lento, provocando um aumento no tempo necessário para atingir o equilíbrio (TAVLIEVA et al., 2013).

\section{CONCLUSÃO}

Os experimentos realizados ao longo do trabalho e as discussões dos resultados obtidos permitiram indicaram que em pHs ácidos tem-se uma melhor remoção do corante e que a melhor condição experimental foi $\mathrm{pH}$ de 2,0, concentração de corante de $25 \mathrm{mg} . \mathrm{L}^{-1}$, agitação de $150 \mathrm{rpm}$, temperatura de $50^{\circ} \mathrm{C}$ e uma concentração de biomassa de 0.5 g gerando uma taxa de remoção de $91,78 \%$.

De acordo com o planejamento experimental a temperatura e agitação apesar de não interferirem de forma isolada, juntas interferem significativamente na capacidade biossortiva. $\mathrm{O}$ mecanismo de remoção se deu com os grupos funcionais presentes na parede celular do Aspergillus niger e na superfície do capim elefante, veículo onde o micro-organismo teve crescimento. Sendo assim a utilização de biomassa fúngica associada a um vegetal pode se tornar uma nova alternativa como biossorvente para tratamento de efluentes têxteis.

\section{REFERÊNCIAS}

BISHNOI, N. R.; GARIMA, A. Fungus - an alternative for bioremediation of heavy metal containing wastewater: a review. Journal of Scientific \& Industrial Research, v. 64, n. 2, p. 93-100, 2005.

BRASIL. Leis, decretos etc. Resolução Conama n 430, de 13 de maio de 2011. Dispõe sobre as condições e padrões de lançamento de efluentes, complementa e altera a Resolução $n^{0} 357$, de 17 de março de 2005, do Conselho Nacional do Meio Ambiente-CONAMA. Disponível em: <http://www.mma.gov.br/port/conama/ legiabre.cfm?codlegi=646>. Acesso em: 24 fev. 2016.

CARDOSO, N. F.; LIMA, E. C.; PINTO, I. S.; AMAVISCA, C. V.; ROYER, B.; PINTO, R. B.; PEREIRA, S. F. Application of cupuassul sheel as biosorbent for the removal of textile dyes from aqueous solution. Journal Environmental Management, v. 92, n. 4, p. 1237-1247, 2011. Disponível em: <http://dx.doi.org/10.1016/ j.jenvman.2010.12.010>. Acesso em: 24 fev. 2016.

DALLAGO, R. M.; SMANIOTTO, A. Resíduos sólidos de curtumes como biossorvente para remoção de corantes em meio aquoso. Química Nova, v. 28, n. 3, p. 433-437, 2005. Disponível em: <http://dx.doi.org/10.1590/S0100-40422005000300013>. Acesso em: 24 fev. 2016. 
KUMAR, R.; BARAKAT, M. A. Decolourization of hazardous brilliant green from aqueous solution using binary oxidized cactus fruit peel. Chemical Engineering Journal, v. 226, p. 377-383, 2013. Disponível em: <http://dx.doi.org/10.1016/ j.cej.2013.04.063>. Acesso em: 24 fev. 2016.

KUNZ, A.; PERALTA-ZAMORA, P.; MORAES, S. G.; DURAN, N. Novas tendências no tratamento de efluentes têxteis. Química Nova, v. 25, n. 1, p. 78-82, 2002. Disponível em: <http://dx.doi.org/10.1590/S0100-40422002000100014>. Acesso em: 24 fev. 2016.

KYZAS, G.; LAZARIDIS, N. K.; MITROPOULOS, A. Removal of dyes from aqueous solutions with untreated coffee residues as potential low-cost adsorbents: equilibrium, reuse and thermodynamic approach. Chemical Engineering Journal, v. 189/190, p. 148-159, 2012. Disponível em: <http://dx.doi.org/10.1016/j.cej.2012.02.045>. Acesso em: 24 fev. 2016.

NETPRADIT, S.; THIRAVETYAN, P.; TOWPRAYOON, S. Adsorption of three azo reactive dyes by metal hydroxide sludge: effect of temperature, $\mathrm{pH}$, and electrolytes. Journal of Colloid and Interface Science, v. 270, p. 255-261, 2004. Disponível em: <http://dx.doi.org/10.1016/j.jcis.2003.08.073>. Acesso em: 24 fev. 2016.

SRINIVASAN, A; VIRARAGHAVAN, T. Decolorization of dye wastewaters by biosorbents: a review. Journal of Environmental Management, v. 91, p. 1915-1929, 2010. Disponível em: <http://dx.doi.org/10.1016/j.jenvman.2010.05.003>. Acesso em: 24 fev. 2016.

TAVLIEVA, M. P., GENIEVA, S. D., GEORGIEVA, V. G., VLAEV, L. T. Kinetic study of brilliant green adsorption from aqueous solution onto white rice husk ash. Journal of Colloid and Interface Science, v. 409, p. 112-122, 2013. Disponível em: <http://dx.doi.org/10.1016/j.jcis.2013.07.052>. Acesso em: 24 fev. 2016. 